\title{
Diagnosing sarcopenia and myosteatosis based on chest computed tomography images in healthy Chinese adults
}

Lingling Tan ${ }^{1 \dagger}$, Guiyi $\mathrm{Ji}^{2 \dagger}$, Ting Bao ${ }^{2}$, Hongbo Fu${ }^{1}$, Ling Yang ${ }^{3^{*}}$ and Ming Yang ${ }^{1,4,5^{*}}$ (D)

\begin{abstract}
Background: Measuring muscle mass and muscle quality based on chest Computed Tomography (CT) images would facilitate sarcopenia and myosteatosis research. We aimed (1) to measure muscle mass and myosteatosis based on chest CT images at the $12^{\text {th }}$ thoracic vertebra level and compare the relevant indicators with whole-body skeletal muscle mass (BSM) and whole-body fat mass (BFM) measured by bioelectrical impedance analysis; and (2) to determine the cut-off points of these indicators for diagnosing sarcopenia or myosteatosis in healthy Chinese adults.
\end{abstract}

Methods: Chest CT images were analyzed using a segmentation software. Skeletal muscle area (SMA), skeletal muscle radiodensity (SMD), and intermuscular adiposity tissue (IMAT) were measured. Skeletal muscle indices (SMIs) and IMAT/SMA ratio were calculated.

Results: We included 569 participants. SMA, SMA/height ${ }^{2}$, and SMA/BMI were strongly and positively correlated with BSM $(r=0.90,0.72$, and 0.69 , respectively, all $p<0.001)$; whereas SMA/weight was moderately and positively correlated with BSM $(r=0.38, p<0.001)$. IMAT and IMAT/SMA were strongly and positively correlated with BFM $(r=0.67$ and 0.58 , respectively, both $p<0.001)$. SMD was moderately and negatively correlated with BFM $(r=-0.40, p<0.001)$. We suggest SMA/height ${ }^{2}\left(<25.75 \mathrm{~cm}^{2} / \mathrm{m}^{2}\right.$ in men and $<20.16 \mathrm{~cm}^{2} / \mathrm{m}^{2}$ in women) for diagnosing sarcopenia and SMD $\left(<37.42 \mathrm{HU}\right.$ in men and $<33.17 \mathrm{HU}$ in women) or IMAT $\left(>8.72 \mathrm{~cm}^{2}\right.$ in men and $>4.58 \mathrm{~cm}^{2}$ in women) for diagnosing myosteatosis.

Conclusions: Muscle mass indicators (SMA and SMIs) and muscle quality indicators (SMD, IMAT, and IMAT/SMA) measured by chest CT images are valuable for diagnosing sarcopenia and myosteatosis, respectively.

Keywords: Muscle wasting, Muscle depletion, Skeletal muscle mass, Computed Tomography, Fat infiltration

*Correspondence: yangling6657@163.com; yangmier@gmail.com

'Lingling Tan and Guiyi Ji contribute equally to this work

${ }^{1}$ Center of Gerontology and Geriatrics, West China Hospital, Sichuan

University, Chengdu, Sichuan, China

${ }^{3}$ Outpatient Department, West China Hospital, Sichuan University, No 37

Guoxue Lane, Chengdu 610041, Sichuan, China

Full list of author information is available at the end of the article

\section{Key points}

- Chest CT images can be used to assess muscle quantity and quality.

- Sarcopenia can be determined using T12 SMA and T12 SMA/height ${ }^{2}$.

- Myosteatosis can be determined using T12 SMD and T12 IMAT. 


\section{Background}

Sarcopenia originally refers to the age-related loss of muscle mass [1]. It has been related to many adverse health outcomes, such as risk of falls, cardiovascular diseases, depression, functional disability, poor quality of life, and even death, in different populations [2-4]. Recently, the updated version of the European Working Group on Sarcopenia in Older People (EWGSOP2), the most widely used sarcopenia guideline, emphasized that muscle quality played an important role in the defining feature of sarcopenia [3].

Muscle quality refers to "micro- and macroscopic changes in muscle architecture and composition" [3]. Fat infiltration in skeletal muscle, also known as myosteatosis, is one of the most widely used indicators for assessing muscle quality [5]. Myosteatosis includes three components: (1) intramyocellular lipids (IMCL); (2) intramuscular adipose tissue (IntraMAT), the extracellular fat within an individual muscle; and (3) intermuscular adipose tissue (IMAT), the extracellular fat beneath the fascia and between muscle groups [5]. Myosteatosis was also found to be associated with adverse outcomes, such as muscle weakness, functional disability, and death [5-10].

Both Magnetic resonance imaging (MRI) and Computed tomography (CT) are suggested to measure muscle mass and myosteatosis [3]. CT is a promising imaging tool for estimating muscle mass via measuring skeletal muscle cross-sectional area (SMA). Additionally, CT can identify myosteatosis not only by directly measuring IMAT but also by indirectly estimating IntraMAT and IMCL via measuring skeletal muscle radiodensity (SMD) expressed in Hounsfield Unit (HU) [5]. The lower the HU, the lower the radiodensity, and the higher the degree of IntraMAT and IMCL [5]. Evaluating whole-body muscle mass and myosteatosis through a whole-body CT scan is supposed to be accurate and reliable, but this is not practical due to high cost and radiation exposure. A more practical method is to measure muscle mass and myosteatosis based on clinically acquired CT scans for the management of other diseases [11]. Therefore, choosing an optimal level of CT axial images to represent whole-body muscle mass or myosteatosis becomes crucial [12]. The $3^{\text {rd }}$ lumbar vertebra (L3) is the commonly used CT level for measuring muscle mass and myosteatosis. However, abdominal CT is not commonly used in clinical practice. In contrast, chest $\mathrm{CT}$ is far more widely used in clinical practice. Therefore, if muscle mass and myosteatosis can be evaluated via chest CT images, the sarcopenia and myosteatosis research based on CT images would be significantly facilitated.

A recent study found a strong correlation between muscle mass measured by chest $\mathrm{CT}$ at the $12^{\text {th }}$ thoracic vertebra (T12) and that measured by abdominal $\mathrm{CT}$ at the L3 level $(r=0.724, p<0.001)$ [13]. Another recent study in JAMA surgery found that CT-defined sarcopenia at the T12 level was associated with one-year mortality in older trauma patients [14]. However, there is currently no study assessing myosteatosis based on chest CT images. Thus, we conducted this study (1) to measure muscle mass and myosteatosis based on chest $\mathrm{CT}$ images at the T12 level and to compare the relevant indicators with whole-body muscle mass and fat mass measured by bioelectrical impedance analysis (BIA); and (2) to determine the cut-off points of these indicators for diagnosing sarcopenia and myosteatosis in a study population of healthy Chinese adults.

\section{Materials and methods \\ Study population}

From August 2019 to July 2020, we continuously invited healthy adults (aged 18 years and older) who received routine health examinations in West China Hospital, Sichuan University, to participate in this study. The exclusion criteria were: (1) individuals who did not receive a chest CT scan; (2) individuals with chronic diseases, such as hypertension, diabetes, coronary heart disease, stroke, chronic respiratory diseases, liver diseases, gastrointestinal diseases, and any type of tumor; (3) individuals with low-quality CT images or had any anatomical distortion such as chest wall edema or loss of any muscle mass area on CT images; (4) individuals with planted pacemaker; and (5) individuals with visible edema. The clinical information collection, anthropometry measurements, and blood samplings were performed on the same day by trained nurses. The study protocol was approved by the Biomedical Ethics Committee of West China Hospital, Sichuan University. All participants signed the written informed consent.

\section{Measurement of anthropometry and laboratory parameters}

The following information was collected from the Electronic Health Record System: age, sex, smoking status, and alcohol drinking status. Waist circumference (WC) was measured at the mid-point between the costal inferior border and the iliac crest in a horizontal plane using a flexible rule to the nearest of $0.1 \mathrm{~cm}$. Body height and weight were measured using an automatic body scale (Sonka Co., Ltd, Shenzhen, China) to the nearest of $0.1 \mathrm{~cm}$ and $0.1 \mathrm{~kg}$, respectively. Body mass index (BMI) was calculated using the equation: $\mathrm{BMI}=$ weight $(\mathrm{kg}) /$ height $^{2}\left(\mathrm{~m}^{2}\right)$.

After at least $8 \mathrm{~h}$ fasting, early morning blood was drawn from an antecubital vein in the arm of each participant. Levels of fasting total cholesterol, triglycerides, high-density lipoprotein-cholesterol (HDL-C), 
and low-density lipoprotein-cholesterol (LDL-C) were tested using the enzymatic colorimetric method with the Toshiba 200FR Neo analyzer (Toshiba Medical System Co., Ltd., Tokyo, Japan).

\section{Measurement of body composition based on $\mathrm{CT}$ images}

Chest CT scans were completed on the same day of anthropometry measurements for each participant using a 16-slice spiral CT scanner (Brilliance; Philips Healthcare, Ohio, USA) with a 5-mm slice thickness. Acquisition parameters were as follows: $100-140 \mathrm{kV}$, variable $\mathrm{mAs}$ based on the patient's body size, and detector collimation of $0.75-1.5 \mathrm{~mm}$.

Unenhanced cross-sectional CT images at the T12 level were analyzed using a dedicated segmentation software (Mimics version 21.0; Materialise, Leuven, Belgium). On a single CT image, skeletal muscle area (SMA) was segmented according to the widely accepted muscle tissues thresholds ( -29 to $150 \mathrm{HU}$ ) [15], including erector spinae, latissimus dorsi, rectus abdominis, obliquus externus, internus abdominis, and internal and external intercostal muscles. The mean of skeletal muscle radiodensity (SMD) of T12 SMA was automatically calculated by Mimics software. The lower the SMD, the higher the degree of myosteatosis. Furthermore, IMAT was segmented according to the widely accepted fat tissue thresholds $(-30$ to $-190 \mathrm{HU})$ [10]. Myosteatosis severity increases with increased IMAT. A trained researcher (L.T.) who was blinded to the participants' clinical information segmented all CT images, and another researcher (G.J.) reviewed the segmented images.

According to previous studies [16, 17], we divided SMA by body height squared $\left(\mathrm{m}^{2}\right)$, body weight $(\mathrm{kg})$, and BMI $\left(\mathrm{kg} / \mathrm{m}^{2}\right)$, respectively, to adjust for the impact of body size on SMA. The height-, weight-, and BMI-adjusted SMAs were collectively known as skeletal muscle indices (SMIs). As reported previously [18], IMAT/SMA ratio was also calculated using the equation: IMAT/SMA ratio $=\operatorname{IMAT}\left(\mathrm{cm}^{2}\right) /$ SMA $\left(\mathrm{cm}^{2}\right) \times 100 \%$.

\section{Body composition measurements based on BIA}

Whole-body skeletal muscle mass (BSM) and wholebody fat mass (BFM) were estimated by segmental multifrequency BIA (InBody 770, Biospace Co., Ltd., Korea). After at least $8 \mathrm{~h}$ fasting, participants were asked to stand on the platform of the BIA device barefoot with their feet on the electrode and to grasp the handles of the device with their fingers directly contacting with the electrodes. Then, they were asked to stand still for $1 \mathrm{~min}$ with their elbows fully extended, and their shoulder abducted to approximately $30^{\circ}$. The device measured the bioimpedance of the participant's body and estimated BSM and BFM automatically.

\section{Statistical analysis}

Continuous data are presented as mean and standard deviation (SD) or median and interquartile range where appropriate; whereas categorical data are presented as number and percentage. The differences between groups were compared using independent samples t-test or Mann-Whitney $U$ test for the continuous variables with normal or abnormal distribution, respectively. The distributions of SMA, SMD, IMAT, and IMAT/SMA in men and women were presented in density plots.

Due to the significant differences in SMA, SMIs (SMA/ height ${ }^{2}$, SMA/weight, and SMA/BMI), SMD, and IMAT between men and women, we stratified the data by sex. Pearson's correlation coefficients ( $r$ ) were calculated to explore the correlations of SMA and SMIs with BSM. Spearman's rank correlation coefficients $(\rho)$ were calculated to explore the correlations of SMD, IMAT, and IMAT/SMA with BFM. We also used scatter plots and linear models to examine the correlation between SMA and BSM and the correlations of SMD and IMAT with BFM. The correlation coefficients are considered as high, moderate, or low when $r$ (or $\rho$ ) is $>0.5,0.3-0.5$, or $<0.3$, respectively [19].

We defined the T-score for SMA and SMIs by calculating the difference between the individual's measured SMA (or SMIs) and the corresponding means of healthy young adults (aged 18 to 40 years). The equation for T-score calculation is as follows: $\mathrm{T}$-score $=$ (individual's value-young adults' mean value)/young adults' SD value. According to the EWGSOP2 [3], individuals with an SMA (or SMIs) less than the sex-specific mean values of SMA (or SMIs) at the point of T-score $=-2.0$ in the young reference group were considered to have sarcopenia. As previously reported $[17,20]$, we also provided the sex-specific mean values of SMA (or SMIs) at the point of $\mathrm{T}$-score $=-1.0$ or -2.5 in the young reference group as the alternative cut-off points of sarcopenia.

Because SMD, IMAT, and IMAT/SMA were of abnormal distribution, SMD-defined myosteatosis was determined when the individual's SMD was less than the sex-specific fifth percentile (p5) cut-off points of SMD in the young reference group. The sex-specific first percentile (p1) and $10^{\text {th }}$ percentile ( $\left.\mathrm{p} 10\right)$ cut-off points of SMD were also reported. Similarly, IMAT-defined myosteatosis was determined when the individual's IMAT was more than the sex-specific $95^{\text {th }}$ percentile (p95) cut-off points of IMAT in the young reference group. The sexspecific $90^{\text {th }}$ percentile $(\mathrm{p} 90)$ and $99^{\text {th }}$ percentile $(\mathrm{p} 99)$ cut-off points of IMAT were also reported.

All statistical analyses were performed in SPSS software 26.0 (IBM SPSS Inc., New York, US) and R version 3.5.1(R Foundation for Statistical Computing, Vienna, Austria). A $p$ value $<0.05$ indicates statistical significance. 


\section{Results}

\section{Study population}

A total of 569 subjects (359 men and 210 women) were included in this study. Table 1 shows the baseline characteristics of the total study population and the young reference group. Not surprisingly, the BSM, BFM, SMA, SMIs, SMD, and IMAT were significantly higher in men than in women. However, there is no significant difference among men and women concerning IMAT/SMA. The density distributions of SMA, SMD, IMAT, and IMAT/SMA of men and women are shown in Figs. 1, 2.

\section{Correlation of body composition indicators measured by $\mathrm{CT}$ and BIA}

Figure 3A shows the correlations (Pearson's r) of SMA and SMIs measured by CT and BSM measured by
BIA; whereas Fig. 3B shows the correlations (Spearman's $\rho$ ) of SMD, IMAT, and IMAT/SMA measured by $\mathrm{CT}$ and BFM measured by BIA. As shown in Fig. 3A, SMA, SMA/height ${ }^{2}$, and SMA/BMI were strongly and positively correlated with BSM $(r=0.90,0.72$, and 0.69 , respectively, all $p<0.001$ ); whereas SMA/weight was moderately and positively correlated with BSM $(r=0.38, p<0.001)$. These findings indicated that SMA, SMA/height ${ }^{2}$, and SMA/BMI were the preferred indicators for estimating BSM.

Furthermore, IMAT and IMAT/SMA were strongly and positively correlated with BFM $(r=0.67$ and 0.58 , respectively, both $p<0.001)$, indicating that both IMAT and IMAT/SMA were the preferred indicators for estimating IMAT-defined myosteatosis. Last, SMD was moderately and negatively correlated with BFM $(r=-0.40, p<0.001)$.

Table 1 Baseline characteristics of the study population and the young adults

\begin{tabular}{|c|c|c|c|c|c|c|}
\hline & \multicolumn{3}{|c|}{ Whole Study Population } & \multicolumn{3}{|c|}{ Young reference group } \\
\hline & Men $(n=359)$ & Women $(n=210)$ & $\mathbf{p}$ & Men $(n=142)$ & Women $(n=102)$ & $\mathbf{p}$ \\
\hline Age (years) & $43.15 \pm 10.67$ & $41.47 \pm 11.03$ & 0.0736 & $32.8 \pm 5.0$ & $31.9 \pm 4.8$ & 0.1743 \\
\hline \multicolumn{7}{|l|}{ Smoking status (\%) } \\
\hline Never & $167(46.52 \%)$ & $202(96.19 \%)$ & $<0.0001$ & $73(51.41 \%)$ & $96(94.12 \%)$ & Fisher $<0.0001$ \\
\hline Ex-smokers & $22(6.13 \%)$ & $0(0.00 \%)$ & & $4(2.82 \%)$ & $0(0.00 \%)$ & \\
\hline Current smokers & $163(45.40 \%)$ & $7(3.33 \%)$ & & $64(45.07 \%)$ & $6(5.88 \%)$ & \\
\hline Miss & $7(1.95 \%)$ & $1(0.48 \%)$ & & $1(0.70 \%)$ & $0(0.00 \%)$ & \\
\hline \multicolumn{7}{|l|}{ Alcohol drinking status (\%) } \\
\hline Never & $66(18.38 \%)$ & $162(77.14 \%)$ & ${ }^{*}$ Fisher < 0.0001 & $26(18.31 \%)$ & $74(72.55 \%)$ & Fisher $<0.0001$ \\
\hline Ex-drinkers & $3(0.84 \%)$ & $0(0.00 \%)$ & & $1(0.70 \%)$ & $0(0.0 \%)$ & \\
\hline Current drinkers & $283(78.83 \%)$ & $47(22.38 \%)$ & & $114(80.28 \%)$ & $28(27.45 \%)$ & \\
\hline Miss & $7(1.95 \%)$ & $1(0.48 \%)$ & & $1(0.70 \%)$ & $0(0.0 \%)$ & \\
\hline Height (cm) & $168.4 \pm 6.2$ & $158.0 \pm 5.8$ & $<0.0001$ & $171.3 \pm 5.6$ & $160.5 \pm 5.6$ & $<0.0001$ \\
\hline Weight (kg) & $69.9 \pm 10.1$ & $53.7 \pm 6.7$ & $<0.0001$ & $71.7 \pm 10.3$ & $53.4 \pm 7.0$ & $<0.0001$ \\
\hline $\mathrm{BMI}\left(\mathrm{kg} / \mathrm{m}^{2}\right)$ & $24.6 \pm 3.0$ & $21.6 \pm 2.6$ & $<0.0001$ & $24.4 \pm 3.2$ & $20.7 \pm 2.4$ & $<0.0001$ \\
\hline \multicolumn{7}{|c|}{ Body composition according to BIA } \\
\hline $\operatorname{BSM}(k g)$ & $29.09 \pm 3.78$ & $20.23 \pm 2.38$ & $<0.0001$ & $30.22 \pm 3.64$ & $20.57 \pm 2.58$ & $<0.0001$ \\
\hline BSM/height ${ }^{2}\left(\mathrm{~kg} / \mathrm{m}^{2}\right)$ & $10.24 \pm 0.98$ & $8.10 \pm 0.72$ & $<0.0001$ & $10.30 \pm 1.01$ & $7.97 \pm 0.78$ & $<0.0001$ \\
\hline $\mathrm{BFM}(\mathrm{kg})$ & $18.26 \pm 5.59$ & $16.50 \pm 4.50$ & 0.0001 & $18.17 \pm 6.08$ & $15.57 \pm 4.13$ & 0.0002 \\
\hline BFM/height ${ }^{2}\left(\mathrm{~kg} / \mathrm{m}^{2}\right)$ & $6.45 \pm 1.96$ & $6.64 \pm 1.91$ & 0.2607 & $6.22 \pm 2.11$ & $6.06 \pm 1.62$ & 0.5226 \\
\hline \multicolumn{7}{|c|}{ Body composition according to CT image } \\
\hline $\mathrm{SMA}\left(\mathrm{cm}^{2}\right)$ & $105.4 \pm 16.22$ & $69.78 \pm 9.84$ & $<0.0001$ & $107.9 \pm 16.1$ & $71.3 \pm 10.5$ & $<0.0001$ \\
\hline SMA/height ${ }^{2}\left(\mathrm{~cm}^{2} / \mathrm{m}^{2}\right)$ & $37.11 \pm 5.51$ & $27.95 \pm 3.77$ & $<0.0001$ & $36.83 \pm 5.54$ & $27.69 \pm 3.76$ & $<0.0001$ \\
\hline SMA/weight $\left(\mathrm{cm}^{2} / \mathrm{kg}\right)$ & $1.51 \pm 0.18$ & $1.31 \pm 0.17$ & $<0.0001$ & $1.51 \pm 0.17$ & $1.34 \pm 0.18$ & $<0.0001$ \\
\hline $\mathrm{SMA} / \mathrm{BMI}\left(\mathrm{cm}^{2} / \mathrm{kg} / \mathrm{m}^{2}\right)$ & $4.29 \pm 0.54$ & $3.26 \pm 0.50$ & $<0.0001$ & $4.44 \pm 0.53$ & $3.47 \pm 0.52$ & $<0.0001$ \\
\hline $\mathrm{SMD}(\mathrm{HU})^{*}$ & $41.46(6.42)$ & $39.52(6.97)$ & $<0.0001$ & $43.66(5.92)$ & $41.73(6.33)$ & $<0.0001$ \\
\hline $\operatorname{IMAT}\left(\mathrm{cm}^{2}\right)^{*}$ & $3.14(3.52)$ & $2.54(2.75)$ & $<0.0001$ & $2.54(2.78)$ & $1.82(2.34)$ & 0.002 \\
\hline IMAT/SMA (\%) & $3.21(3.39)$ & $3.47(4.12)$ & 0.247 & $2.60(2.58)$ & $2.47(2.97)$ & 0.832 \\
\hline
\end{tabular}

Data presented as $\mathrm{n}$ (percentage) or mean (standard deviation) if not specified

BMI: body mass index; BFM: body fat mass; BSM: body skeletal muscle; IMAT: intermuscular adipose tissue; SMA: skeletal muscle area; SMD: skeletal muscle radiodensity

* Data presented as median (interquartile range) 

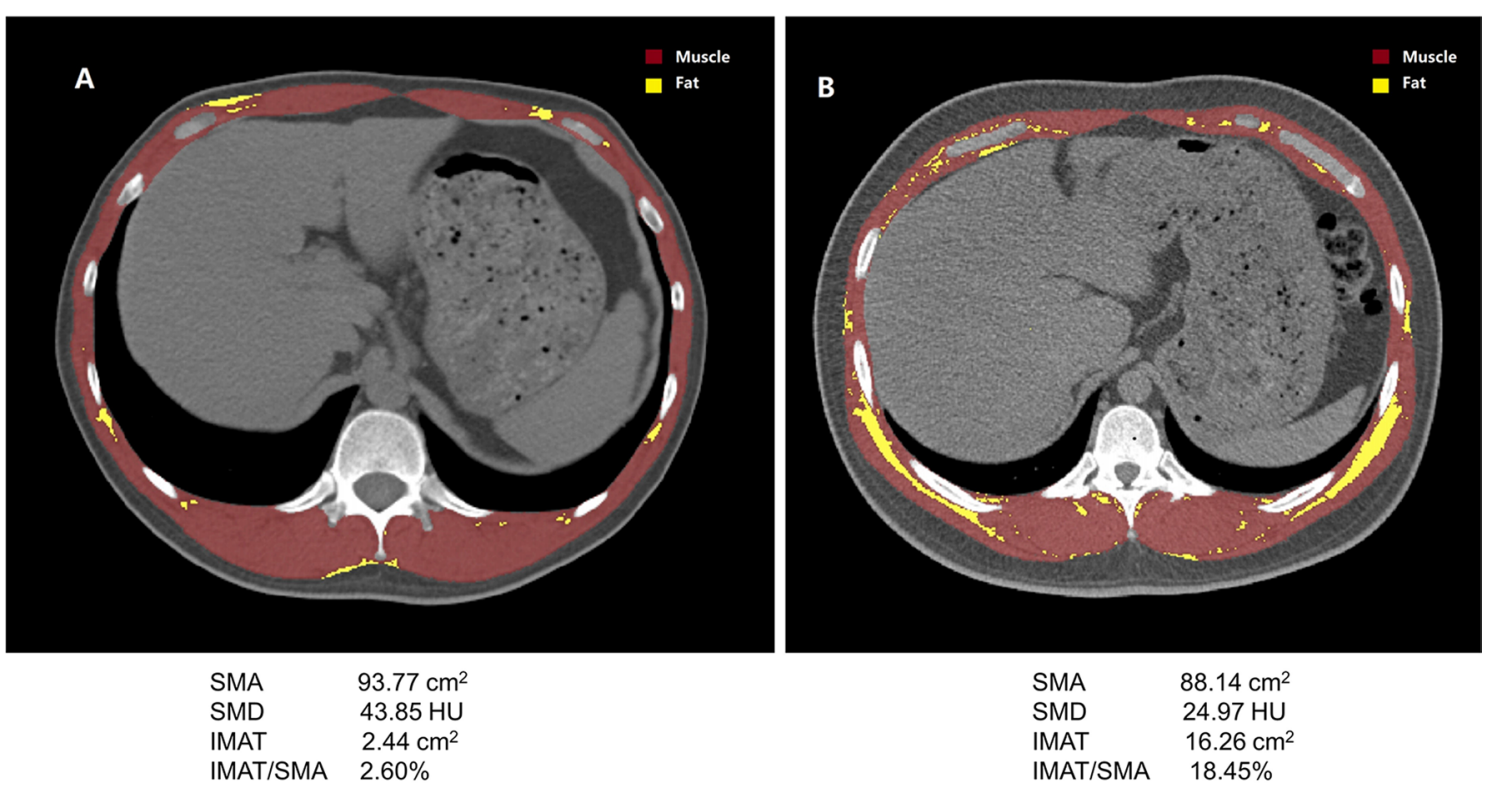

Fig. 1 Cross-sectional CT images at the T12 level used for the quantification of muscle mass and fat mass in a 39-year-old man (A) and a 62-year-old woman (B)

Figure 4 shows the scatter plots and linear regression lines of SMA with BSM (A), IMAT with BFM (B), and SMD with BFM (C) in men and women.

\section{Reference values for defining sarcopenia and myosteatosis by SMA, SMIs, SMD, IMAT, and IMAT/SMA}

Table 2 shows the sex-specific cut-off points of SMA and SMIs equivalent to T-score $-1.0,-2.0$, and -2.5 ; SMD equivalent to $\mathrm{p} 1, \mathrm{p} 5$, and $\mathrm{p} 10$; and IMAT and IMAT/SMA equivalent to p90, p95, and p99 in the young reference group. When T-score $<-2.0$ was used to set the cut-off point for defining sarcopenia, the sex-specific cut-off points of SMA and SMA/ height ${ }^{2}$ were $<75.67 \mathrm{~cm}^{2}$ and $<25.75 \mathrm{~cm}^{2} / \mathrm{m}^{2}$ in men, and $<50.39 \mathrm{~cm}^{2}$ and $<20.16 \mathrm{~cm}^{2} / \mathrm{m}^{2}$ in women, respectively. When $\mathrm{p} 5$ was used to set the cut-off point for determining SMD-defined myosteatosis, the cut-off points were $<37.42 \mathrm{HU}$ and $<33.17 \mathrm{HU}$ in men and women, respectively. When p95 was used to set the cut-off point for determining IMAT-defined myosteatosis, the sex-specific cut-off points of IMAT and IMAT/SMA were $>8.72 \mathrm{~cm}^{2}$ and $>7.51 \%$ in men, respectively, and $>4.58 \mathrm{~cm}^{2}$ and $>6.83 \%$ in women, respectively.

\section{Discussion}

This study demonstrated that T12 SMA and T12 SMA/ height ${ }^{2}$ were strongly correlated with BSM; T12 IMAT was strongly correlated with BFM; whereas T12 SMD was moderately correlated with BFM. These findings imply that T12 SMA and T12 SMA/height ${ }^{2}$ may serve as surrogates for diagnosing sarcopenia while T12 SMD and T12 IMAT may serve as surrogates for determining myosteatosis. To the best of our knowledge, this is the first study to report the cut-off points of T12 SMA (and SMIs) for diagnosing sarcopenia, and the cut-off points of T12 SMD and T12 IMAT for diagnosing myosteatosis in Chinese people.

Only two previous studies reported the cut-off points of T12 SMA (and SMIs) measured by chest CT for diagnosing sarcopenia. Both were conducted in Caucasians. Based on a healthy US population, Derstine et al. [20] reported the cut-off points of T12 SMA and SMA/height ${ }^{2}$ for defining sarcopenia were $92.3 \mathrm{~cm}^{2}$ and $28.8 \mathrm{~cm}^{2} / \mathrm{m}^{2}$ in men, and $56.1 \mathrm{~cm}^{2}$ and $20.8 \mathrm{~cm}^{2} / \mathrm{m}^{2}$ in women, respectively. Based on the Michigan Kidney Donor Candidate Population, the same team reported the cut-off points of T12SMA and SMA/height ${ }^{2}$ for defining sarcopenia were $91.5 \mathrm{~cm}^{2}$ and $28.7 \mathrm{~cm}^{2} / \mathrm{m}^{2}$ in men, and $55.9 \mathrm{~cm}^{2}$ and 20.6 $\mathrm{cm}^{2} / \mathrm{m}^{2}$ in women, respectively [21]. As expected, the T12 SMA in the US population was higher than that in our study population in both men and women. However, after adjusting for body size, the T12 SMA/height ${ }^{2}$ was very similar across the three study populations. This finding supports the utility of T12 SMA/height ${ }^{2}$ (rather than SMA) to define sarcopenia to facilitate the comparability between studies.

Notably, there remains an unsolved controversy regarding the definition of sarcopenia [11]. In brief, most 


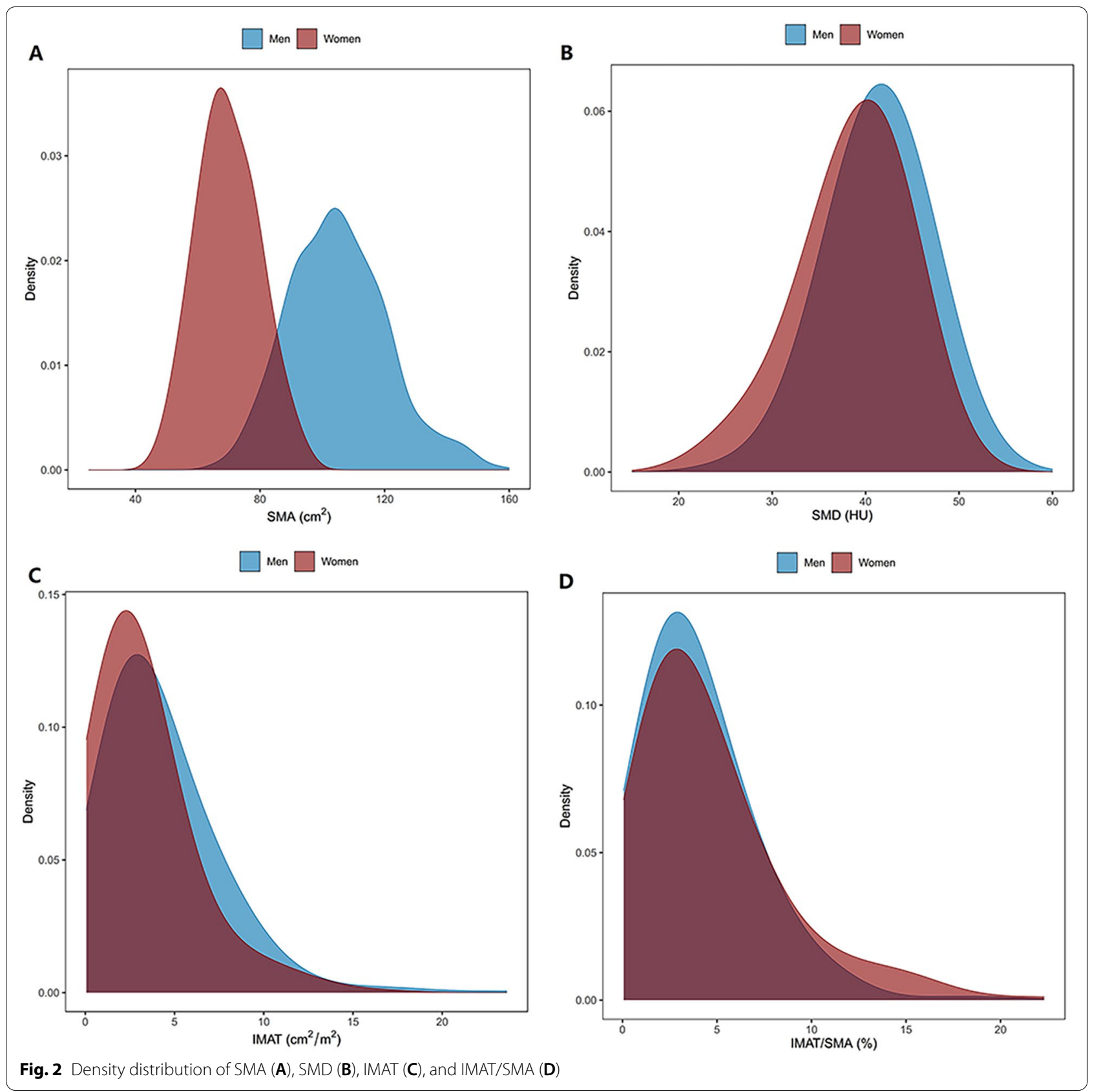

researchers in the field of oncology and surgery define sarcopenia as low muscle mass per se [22]. For example, the North American Expert Opinion Statement on Sarcopenia in Liver Transplantation recommends defining sarcopenia "using only muscle mass" [23]. In contrast, in the field of geriatrics and gerontology, a consensus has been achieved that the definition of sarcopenia should include not only low muscle mass but also low muscle strength and/or low physical performance [2, 24]. Traditionally, CT-defined sarcopenia was widely studied in oncology and surgery because opportunistic CT images could easily be obtained from patients with cancer or having surgery [11]. However, studies regarding CTdefined sarcopenia in older adults are emerging $[25,26]$.

Myosteatosis, as an important indicator of muscle quality, has increasingly been attracting researchers' attention in the field of skeletal muscle aging [5]. The diagnosis methods of myosteatosis have not been established yet; however, IMAT and SMD were the two most frequently used indicators to define myosteatosis in the literature 

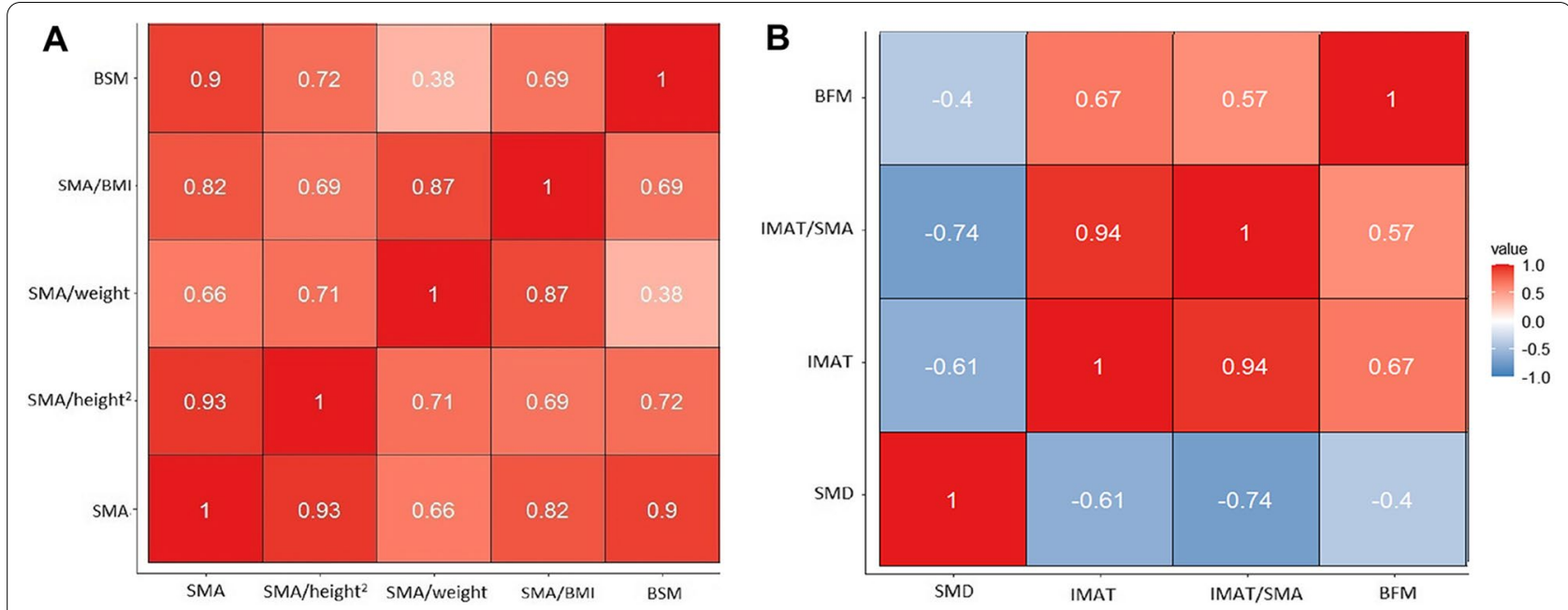

Fig. 3 The correlations (Pearson's r) of SMA and SMIs measured by CT and BSM measured by BIA (A) and the correlations (Spearman's $\rho$ ) of SMD, IMAT, and IMAT/SMA measured by CT and BFM measured by BIA (B)

[5]. According to a recent systematic review, among the 125 included studies, $82(65.6 \%)$ and $27(21.6 \%)$ studies used IMAT and SMD to define myosteatosis, respectively [11]. Besides, the abdomen and thigh were the most frequently scanned body positions in the literature. In this same systematic review, $61(48.8 \%)$ and $48(38.4 \%)$ studies used abdominal CT images and thigh CT images, respectively. However, no study defined myosteatosis based on chest CT [11]. Our study provided the cut-off points of T12 SMD (men: $<37.42 \mathrm{HU}$; women: $<33.17$ HU) and T12 IMAT (men: $>8.72 \mathrm{~cm}^{2}$; women: $>4.58$ $\mathrm{cm}^{2}$ ) for defining myosteatosis base on chest CT. This method can be used to define myosteatosis in Asian populations. However, these cut-off points need to be further validated in different populations.

Which one is better for defining myosteatosis, SMD or IMAT? There is currently no answer to this question. However, a recent study indicated that SMD (but not IMAT) was independently associated with peak knee extension torques and rates of torque development in older adults [27]. In a retrospective study conducted in ICU patients, higher SMD was significantly associated with lower 6-month mortality; whereas higher IMAT was not significantly associated with higher 6-moth mortality after adjustment for confounders [28]. Furthermore, it has been reported that both SMD and IMAT were associated with mortality in men; but only SMD (not IMAT) was associated with mortality in women [5]. More prospective studies are needed to answer this question.

MRI is supposed to be the gold standard to analyze body composition, especially myosteatosis. MRI is a more sensitive tool for detecting muscle fat diffusion and visualizing anatomical structures than CT [29,
30]. Additionally, MRI can analyze the composition and detailed structure of individual muscles, assisting in the differentiation of edema, fatty infiltration, and fibrosis $[29,30]$. Furthermore, magnetic resonance spectroscope (MRS) can precisely differentiate IMCL, IntraMAT, and IMAT [31]. Both MRI and MRS do not expose patients to radiation. However, MRI and MRS are very expensive and not readily accessible, which limits their use in clinical research and practice as tools to identify sarcopenia and myosteatosis [31].

\section{Clinical implications}

Compared with BIA and dual-energy x-ray absorptiometry (DXA), CT is limited because it exposes the participants to radiation. Therefore, muscle mass and muscle quality measurements are commonly analyzed based on clinically acquired CT scans for the management of other diseases [11]. In clinical practice, chest CT scans are far more frequently used than abdominal CT and thigh CT scans. In China, chest CT scans have been widely used in routine health examinations for screening lung cancer and other diseases in middle-aged and older adults. Therefore, the opportunistic utility of chest CT images would provide a greater opportunity to identify other diseases, such as chronic obstructive pulmonary disease [32] and osteoporosis [33]. Our study implies that the opportunistic utility of chest CT images is also useful for identifying sarcopenia and myosteatosis.

Notably, our study segmented SMA and IMAT in the CT images based on the most widely accepted HU thresholds (SMA: -29 to 150 HU; IMAT: -30 to -190 $\mathrm{HU})$ according to the recent systematic reviews [11, 34]. 

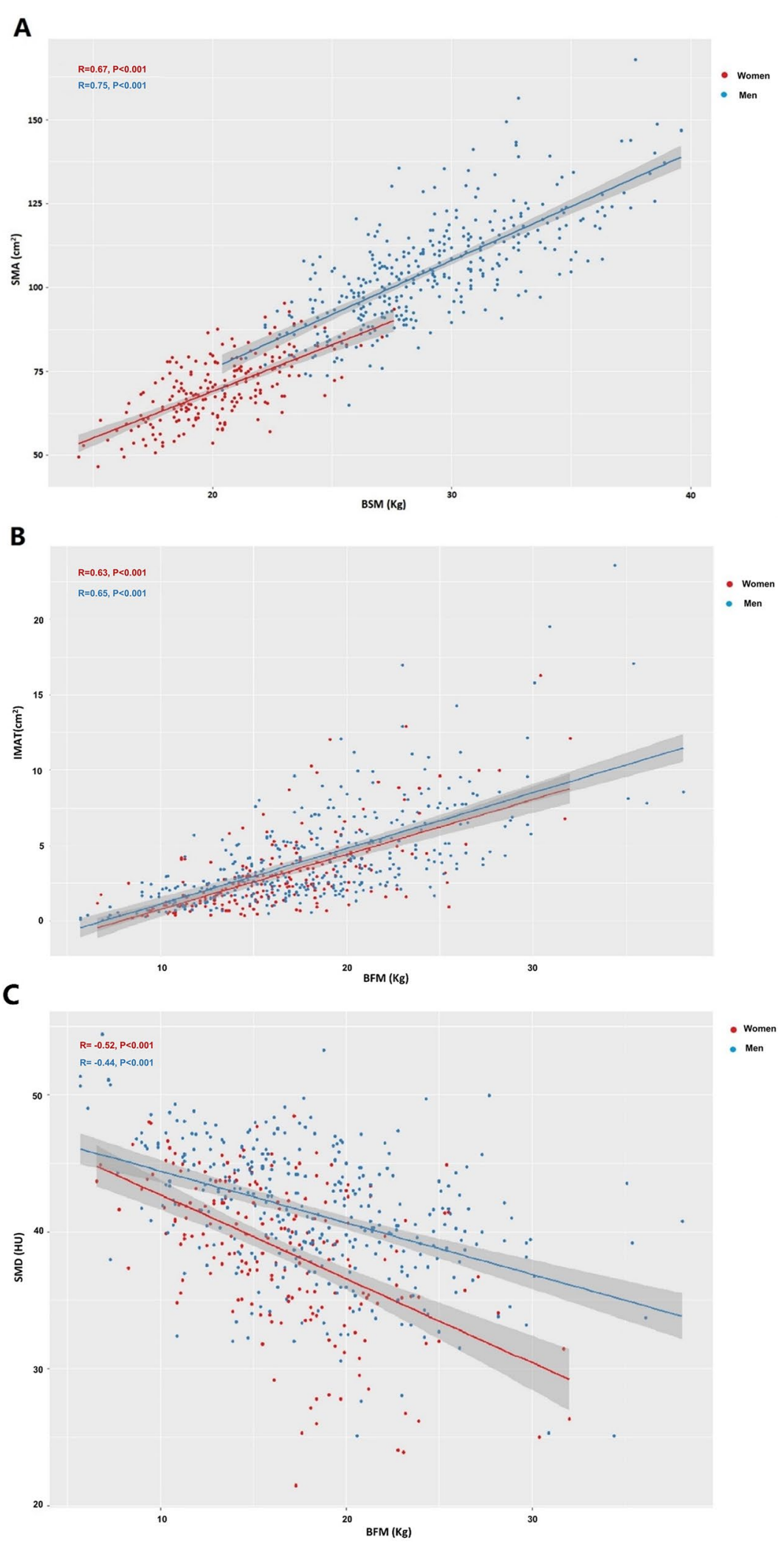

Fig. 4 The scatter plots and linear regression lines of SMA with BSM (A), IMAT with BFM (B), and SMD with BFM (C) in men and women 
Table 2 Reference data from the young reference group (20-40 years) regarding SMA, SMD, IMAT, and their indices

\begin{tabular}{|c|c|c|c|c|c|c|}
\hline & \multicolumn{3}{|l|}{ Men $(n=142)$} & \multicolumn{3}{|c|}{ Women $(n=102)$} \\
\hline & T score $=-1.0$ & $\mathrm{~T}$ score $=-2.0$ & T score $=-2.5$ & T score $=-1.0$ & T score $=-2.0$ & T score $=-2.5$ \\
\hline $\mathrm{SMA}\left(\mathrm{cm}^{2}\right)$ & 91.76 & 75.67 & 67.62 & 60.85 & 50.39 & 45.16 \\
\hline SMA/height ${ }^{2}\left(\mathrm{~cm}^{2} / \mathrm{m}^{2}\right)$ & 31.29 & 25.75 & 22.98 & 23.92 & 20.16 & 18.28 \\
\hline SMA/weight $\left(\mathrm{cm}^{2} / \mathrm{kg}\right)$ & 1.34 & 1.16 & 1.08 & 1.17 & 0.99 & 0.9 \\
\hline \multirow[t]{2}{*}{$\mathrm{SMA} / \mathrm{BMI}\left(\mathrm{cm}^{2} / \mathrm{kg} / \mathrm{m}^{2}\right)$} & 3.91 & 3.37 & 3.11 & 2.95 & 2.43 & 2.17 \\
\hline & p1 & p5 & p10 & p1 & p5 & p10 \\
\hline \multirow[t]{2}{*}{$\mathrm{SMD}(\mathrm{HU})$} & 33.76 & 37.42 & 39.21 & 29.25 & 33.17 & 34.46 \\
\hline & p99 & p95 & p90 & p99 & p95 & p90 \\
\hline IMAT $\left(\mathrm{cm}^{2}\right)$ & 14.35 & 8.72 & 7.17 & 5.28 & 4.58 & 4.01 \\
\hline IMAT/SMA (\%) & 11.19 & 7.51 & 6.27 & 8.99 & 6.83 & 6.19 \\
\hline
\end{tabular}

BMI: body mass index; IMAT: intermuscular adipose tissue area; $\mathrm{P} 1$ : the $1^{\text {st }}$ percentile; $\mathrm{P} 5$ : the $5^{\text {th }}$ percentile; $\mathrm{P} 10$, the $10^{\text {th }}$ percentile; $\mathrm{P} 90$ : the $90^{\text {th }}$ percentile; $\mathrm{P} 95$ : the $95^{\text {th }}$ percentile; P99: the $99^{\text {th }}$ percentile; SMA: skeletal muscle area; SMD: skeletal muscle radiodensity

Therefore, the cut-off points for the muscle mass and muscle quality indicators provided in our study may not be suitable for the studies that apply other HU thresholds to define skeletal muscle mass or IMAT (e.g., 0 to $100 \mathrm{HU}$ to define muscle mass as previously reported).

\section{Limitations}

First, the participants of this study were participants in routine health examinations at the health management center of a tertiary hospital, which is the largest hospital in western China that provides comprehensive health examinations for individuals from western China. Because the participants were voluntarily joined in this study during routine health examinations, the representation of our study population may be limited. Therefore, the generalization of our results to other populations should be cautious. Second, we estimated the BSM and BFM with BIA instead of DXA. However, a recent study found that DXA and segmental multi-frequency BIA (InBody 770, the exact device type that we used in this study) were comparable for estimating BSM and BFM [35]. Third, it remains unclear whether the cut-off points of muscle mass and muscle quality indicators that we proposed in this study can predict outcomes or not. We have conducted a prospective cohort study in patients with advanced non-small-cell lung cancer to validate our results.

\section{Conclusion}

Muscle mass indicators (SMA and SMIs) and muscle quality indicators (SMD, IMAT, and IMAT/SMA) measured by chest CT images at the T12 level could be used for diagnosing sarcopenia and myosteatosis, respectively. We suggest SMA/height ${ }^{2}\left(<25.75 \mathrm{~cm}^{2} / \mathrm{m}^{2}\right.$ in men and $<20.16 \mathrm{~cm}^{2} / \mathrm{m}^{2}$ in women) for diagnosing sarcopenia and SMD $(<37.42 \mathrm{HU}$ in men and $<33.17 \mathrm{HU}$ in women $)$ or
IMAT ( $>8.72 \mathrm{~cm}^{2}$ in men and $>4.58 \mathrm{~cm}^{2}$ in women) for diagnosing myosteatosis in Chinese people. However, the cut-off points of these indicators need to be further validated in different populations.

\section{Abbreviations}

BFM: Whole-body fat mass; BIA: Bioelectrical impedance analysis; BMI: Body mass index; BSM: Whole-body skeletal muscle mass; CT: Computed tomography; EWGSOP2: The updated version of the European Working Group on Sarcopenia in Older People; HDL-C: High-density lipoprotein-cholesterol; HU: Hounsfield Unit; IMAT: Intermuscular adiposity tissue; IMCL: Intramyocellular lipids; IntraMAT: Intramuscular adipose tissue; L3: The $3^{\text {rd }}$ lumbar vertebra; LDL-C: Low-density lipoprotein-cholesterol; SMA: Skeletal muscle area; SMD: Skeletal muscle radiodensity; SMI: Skeletal muscle index; T12: The $12^{\text {th }}$ thoracic vertebra; WC: Waist circumference.

\section{Authors' contributions}

Conception and Design of study: MY; Imaging data analysis: GJ, LT, and HF; Acquisition, analysis, and interpretation of data: All authors; Drafting the manuscript: LT and MY; Critical revision of the manuscript for important intellectual content: All authors; Statistical analysis: TB and LT. All authors read and approved the final manuscript.

\section{Funding}

This work was supported by the K\&D Program of the Sichuan Science and Technology Department (Grant Number: 2020YFS0573).

\section{Availability of data and materials}

The datasets used and/or analyzed during the current study are available from the corresponding author on reasonable request.

\section{Declarations}

Ethics approval and consent to participate

The study protocol was approved by the Biomedical Ethics Committee of West China Hospital, Sichuan University (No. 2019-238). All participants signed the written informed consent.

\section{Consent for publication}

Not applicable.

Competing interests

The authors declare that they have no competing interests. 


\section{Author details}

${ }^{1}$ Center of Gerontology and Geriatrics, West China Hospital, Sichuan University, Chengdu, Sichuan, China. ${ }^{2}$ Health Management Center, West China Hospital, Sichuan University, Chengdu, Sichuan, China. ${ }^{3}$ Outpatient Department, West China Hospital, Sichuan University, No 37 Guoxue Lane, Chengdu 610041, Sichuan, China. ${ }^{4}$ National Clinical Research Center for Geriatrics, West China Hospital, Sichuan University, No 37 Guoxue Lane, Chengdu 610041, Sichuan, China. ${ }^{5}$ Precision Medicine Research Center, West China Hospital, Sichuan University, Chengdu, Sichuan, China.

Received: 26 July 2021 Accepted: 5 October 2021

Published online: 06 November 2021

\section{References}

1. Cruz-Jentoft AJ, Sayer AA (2019) Sarcopenia. Lancet 393(10191):2636-2646

2. Cruz-Jentoft AJ, Bahat G, Bauer J et al (2019) Sarcopenia: revised European consensus on definition and diagnosis. Age Ageing 48(1):16-31

3. Cruz-Jentoft AJ, Baeyens JP, Bauer et al (2010) Sarcopenia: European consensus on definition and diagnosis: Report of the European Working Group on Sarcopenia in Older People. Age Ageing 39(4):412-423

4. Paoloni VSABMMM (2014) Clinical definition of sarcopenia. Clin Cases Min Bone Metab 1(3)

5. Correa-de-Araujo R, Addison O, Miljkovic I et al (2020) Myosteatosis in the context of skeletal muscle function deficit: An interdisciplinary workshop at the national institute on aging. Front Physiol 11:963

6. Lattanzi B, Nardelli S, Pigliacelli A et al (2019) The additive value of sarcopenia, myosteatosis and hepatic encephalopathy in the predictivity of model for end-stage liver disease. Dig Liver Dis 51(11):1508-1512

7. Goodpaster BH, Carlson CL, Visser M et al (2001) Attenuation of skeletal muscle and strength in the elderly: The health $A B C$ study. J Appl Physiol 90(6):2157-2165

8. Visser M, Kritchevsky SB, Goodpaster BH et al (2002) Leg muscle mass and composition in relation to lower extremity performance in men and women aged 70 to 79: The health, aging and body composition study. J Am Geriatr Soc 50(5):897-904

9. Visser M, Goodpaster BH, Kritchevsky SB et al (2005) Muscle mass, muscle strength, and muscle fat infiltration as predictors of incident mobility limitations in well-functioning older persons. J Gerontol A Biol Sci Med Sci 60(3):324-333

10. Lee K, Shin Y, Huh J et al (2019) Recent issues on body composition imaging for Sarcopenia evaluation. Korean J Radiol 20(2):205-217

11. Amini B, Boyle SP, Boutin RD, Lenchik $L$ (2019) Approaches to assessment of muscle mass and myosteatosis on computed tomography: a systematic review. J Gerontol Ser

12. Faron A, Luetkens JA, Schmeel FC, Kuetting DLR, Thomas D, Sprinkart AM (2019) Quantification of fat and skeletal muscle tissue at abdominal computed tomography: associations between single-slice measurements and total compartment volumes. Abdom Radiol (NY) 44(5):1907-1916

13. Nemec U, Heidinger B, Sokas C, Chu L, Eisenberg RL (2017) Diagnosing sarcopenia on thoracic computed tomography: quantitative assessment of skeletal muscle mass in patients undergoing transcatheter aortic valve replacement. Acad Radiol 24(9):1154-1161

14. Kaplan SJ, Zhao KL, Koren M, Bentov I, Reed MJ, Pham TN (2020) Thresholds and mortality associations of paraspinous muscle sarcopenia in older trauma patients. JAMA Surg 155(7):662-664

15. Park J, Gil JR, Shin Y et al (2019) Reliable and robust method for abdominal muscle mass quantification using CT/MRI: An explorative study in healthy subjects. PLoS One 14(9):e0222042

16. Montano-Loza AJ, Angulo P, Meza-Junco J et al (2016) Sarcopenic obesity and myosteatosis are associated with higher mortality in patients with cirrhosis. J Cachexia Sarcopenia Muscle 7(2):126-135

17. Kim EH, Kim KW, Shin Y et al (2020) Reference data and T-scores of lumbar skeletal muscle area and its skeletal muscle indices measured by ct scan in a healthy korean population. J Gerontol A Biol Sci Med Sci 76(2):265-271

18. Perkisas S, Cock AMD, Verhoeven V, Vandewoude M (2017) Intramuscular adipose tissue and the functional components of sarcopenia in hospitalized geriatric patients. Geriatrics 2(1):11

19. Bhullar AS, Anoveros-Barrera A, Dunichand-Hoedl A et al (2020) Lipid is heterogeneously distributed in muscle and associates with low radiodensity in cancer patients. J Cachexia Sarcopenia Muscle 11(3):735-747

20. Derstine BA, Holcombe SA, Ross BE, Wang NC, Su GL, Wang SC (2018) Skeletal muscle cutoff values for sarcopenia diagnosis using T10 to L5 measurements in a healthy US population. Sci Rep 8(1)

21. Derstine BA, Holcombe SA, Goulson RL et al (2018) Quantifying sarcopenia reference values using lumbar and thoracic muscle areas in a healthy population. J Nutr Health Aging 22(1):180-185

22. Daly LE, Prado CM, Ryan AM (2018) A window beneath the skin: how computed tomography assessment of body composition can assist in the identification of hidden wasting conditions in oncology that profoundly impact outcomes. Proc Nutr Soc 77(2):135-151

23. Carey EJ, Lai JC, Sonnenday C et al (2019) A North american expert opinion statement on sarcopenia in liver transplantation. Hepatology 70(5):1816-1829

24. Chen L-K, Woo J, Assantachai P et al (2020) Asian working group for Sarcopenia: 2019 consensus update on Sarcopenia diagnosis and treatment. J Am Med Dir Assoc 21(3):300-307.e302

25. Lenchik L, Lenoir KM, Tan J et al (2018) Opportunistic measurement of skeletal muscle size and muscle attenuation on computed tomography predicts 1-year mortality in medicare patients. J Gerontol Ser 74(7):1063-1069

26. Lenchik L, Barnard R, Boutin RD et al (2020) Automated muscle measurement on chest computed tomography (CT) predicts all-cause mortality in older adults from the national lung screening trial. J Gerontol A Biol Sci Med Sci 76(2):277-285

27. Frank-Wilson AW, Chalhoub D, Fiqueiredo P et al (2018) Associations of quadriceps torque properties with muscle size, attenuation, and intramuscular adipose tissue in older adults. J Gerontol Ser 73(7):931-938

28. Looijaard W, Dekker IM, Stapel SN, Girbes ARJ, Twisk JWR, Straaten HMOV, Weijs PJM (2016) Skeletal muscle quality as assessed by CT-derived skeletal muscle density is associated with 6-month mortality in mechanically ventilated critically ill patients. Crit Care 20(1):386

29. Seabolt LA, Welch EB, Silver HJ (2015) Imaging methods for analyzing body composition in human obesity and cardiometabolic disease. Ann N Y Acad Sci 1353:41-59

30. Gloor M, Fasler S, Fischmann A et al (2011) Quantification of fat infiltration in oculopharyngeal muscular dystrophy: comparison of three MR imaging methods. J Magn Reson Imaging 33(1):203-210

31. Naimo MA, Varanoske AN, Hughes JM, Pasiakos SM (2021) Skeletal muscle quality: a biomarker for assessing physical performance capabilities in young populations. Front Physiol 12:706699.

32. Mets OM, Buckens CFM, Zanen P et al (2011) Identification of chronic obstructive pulmonary disease in lung cancer screening computed tomographic scans. JAMA 306(16):1775-1781

33. Buckens CF, Graaf Yvd, Verkooijen HM et al (2015) Osteoporosis markers on low-dose lung cancer screening chest computed tomography scans predict all-cause mortality. Eur Radiol 25(1):132-139

34. Ahn H, Kim DW, Ko Y et al (2021) Updated systematic review and metaanalysis on diagnostic issues and the prognostic impact of myosteatosis: A new paradigm beyond sarcopenia. Ageing Res Rev 101398

35. Hurt RT, Ebbert JO, Croghan I et al (2020) The comparison of segmental multi-frequency BIA and DXA for estimating lean body mass and percent body fat in an ambulatory population. JPEN J Parenter Enteral Nutr 45(6):1231-1238

\section{Publisher's Note}

Springer Nature remains neutral with regard to jurisdictional claims in published maps and institutional affiliations. 